\title{
Brain solutions for hearing problems during the COVID-19 pandemic and the misery of wearing a mask
}

\author{
Nima Derakhshan ${ }^{1}$ (D) Shekoofeh Yaghmaei ${ }^{2}$ (D)
}

Received: 13 September 2020 / Accepted: 30 October 2020 / Published online: 23 November 2020

(c) Springer-Verlag GmbH Germany, part of Springer Nature 2020

\begin{abstract}
Background In COVID-19 pandemics days, wearing facial mask in public places has become obligatory to prevent the virus spread. In addition to its valuable protection, wearing facial mask can affect verbal communication in an adverse fashion and makes mutual understanding difficult. This happens because the mask eliminates the positive effect of the lip-reading phenomenon in direct communications. The mirror neuron system is responsible for automatic imitation, associative sequence learning, and motor mimicry. This system is a strong candidate justifying an unexpected action described in this article.

Purpose Taking the facial mask off, to help the listener understanding better is a normal reaction. However, unexpectedly, one does the same as the listener when he/she is unable to comprehend the speaker. Herein, we suggest a hypothesis proposing the basic role of Mirror neuron system in this action. Most of the research on these cells have been conducted on monkeys, where the researchers observed that, these neuron discharge pulses both when a monkey performs an action and when it observes another monkey or a person committing the similar action.

Conclusion The driving mechanism of an unanticipated action of taking off mask while listening to a speaker is emphasized in this paper. Herein, we try to clarify how we came up with the idea that mirror neuron system drives a surprising action observed in COVID-19 pandemics days. As a result, we suggest possible clinical studies to verify our hypothesis.
\end{abstract}

Keywords Mirror neuron system · COVID-19 · Facial mask · Communication difficulty

The COVID-19 (coronavirus) outbreak was first identified in December 2019 in Wuhan, China. Later on March 12th 2020, WHO (World Health Organization) announced this outbreak as a pandemic [1]. Thereafter, wearing face masks, social distancing, and frequent hand washing were highly recommended as effective preventive acts. Due to its double duty protection, more than 50 countries have enforced compulsory face mask policies in public areas. However, if used thoughtlessly, the masks can also cause undesired side effects. While masks save lives, they also create social challenges like verbal communication difficulties which are more considerable for people suffering from hearing

Shekoofeh Yaghmaei

yaghmaei.shekoofeh@gmail.com

Nima Derakhshan

nima_med83@yahoo.com

1 Research Center for Neuromodulation and Pain, Shiraz University of Medical Sciences, Shiraz, Iran

2 General Practitioner, Shiraz University of Medical Sciences, Zand St., PO Box: 71348-14336, Shiraz, Iran impairment. In cases that verbal communication cannot be fully effective (e.g. in crowded environments, in areas with high noise level, or for people with hearing disabilities) the brain employs the lip-reading mechanism to improve the communication.

Nowadays, cloth masks that people wear in public areas are major obstacles to lip-reading. Transparent face masks and face shields are available alternatives to cloth face masks providing the possibility of lip-reading. Unfortunately, they do not provide adequate protection from COVID-19 and are not currently approved as a substitute for cloth masks [2].

As wearing non-transparent facial masks has become rather common in pandemic days, whenever an audience cannot understand one's message, the speaker takes off his/her mask to add the lip-reading assistance to the communication. This is a very logical and expected reaction. However, some people take their masks off even when they cannot understand others' meaning which is quite surprising. This is unexpected, because taking off the listener's mask does not have any influence on delivering speech. 
Herein, we support the hypothesis that this reaction is a result of the MNS (mirror neuron system) response.

MNS, a group of neurons in parietal and premotor cortex, were first discovered by Giacomo Rizollatti observing a family of monkeys named Macaques [3]. These neurons discharge not only when the monkey does a particular action, but also when it observes another individual, either monkey or human, doing a similar action (for example eating a banana) [4]. Another example is contagious yawning [5] or body movement during computer games, such as pressing the foot on the ground when braking in racing video games or bending the body in the same direction as in the car turns. All these unconscious reactions are rooted in MNS performance. MNS is required for both understanding the actions of others and arranging own actions [6]. It is believed to encode properties of an observed action and code a connection between action and observation. For instance, some neurons may code motor parameters, others may code goals or even both [7]. Heyes suggests MNS as a key explaining a number of social behaviors such as reading minds and imitation [8].

As one observes that, as a listener, he/she understands the speech better if the speaker takes off his/her mask, he/she (the listener) correlates taking off mask with conversation improvement. MNS also drives him/her to take off the mask even when he/she is a speaker in an ineffective conversation.

Funding None.

Data availability None.

Code availability None.

\section{Compliance with ethical standards}

Conflict of interest The authors declared that they have no conflicts of interest.

\section{References}

1. World Health Organization (2020, march 12) Coronavirus disease (COVID-19) pandemic. https://www.euro.who.int/en/healt h-topics/health-emergencies/coronavirus-covid-19/novel-coron avirus-2019-ncov Accessed March 12, 2020

2. Centers for diseasse control and prevention (Updated 4, Nov, 2020) Considerations for wearing masks. https://www.cdc.gov/ coronavirus/2019-ncov/prevent-getting-sick/cloth-face-cover -guidance.html. Accessed 4 Nov 2020

3. Taghipour M, Derakhshan N (2018) Hypothesis on the origin of dance: mirror neuron system and imitation from movement disorders. World Neurosurg 120:604-604

4. Rizzolatti G, Craighero L (2004) The mirror-neuron system. Annu Rev Neurosci 27:169-192

5. Provine RR (1989) Contagious yawning and infant imitation. Bull Psychon Soc 27(2):125-126

6. Pomper JK, Spadacenta S, Bunjes F, Arnstein D, Giese MA, Thier $P$ (2020) Representation of the observer's predicted outcome value in mirror and non-mirror neurons of macaque F5 ventral premotor cortex. J Neurophysiol 124(3):941-961

7. Oztop E, Kawato M, Arbib MA (2013) Mirror neurons: functions, mechanisms and models. Neurosci Lett 540:43-55

8. Heyes C (2010) Where do mirror neurons come from? Neurosci Biobehav Rev 34(4):575-583

Publisher's Note Springer Nature remains neutral with regard to jurisdictional claims in published maps and institutional affiliations. 